Ciência e Natura, v. 37 Part 2 2015, p. 364-370

\title{
A Chaos- Based Video watermarking in Wavelet Domain
}

\author{
Somayyeh Mohammadi
}

Department of Engineering Payame Noor (PNU) University,Kerman, Iran

\begin{abstract}
A novel video watermarking scheme based on chaotic maps and wavelet transform is proposed in this paper. The algorithm encrypts a logo image as a watermark signal by bit xor'ing the pixel values of logo and a chaotic map, thus the security of the proposed scheme will be increased. In order to enjoy a robust watermarking algorithm, the chaotic watermark signal is embedded into the discrete wavelet transform coefficients of I-frames,. The investigational results demonstrate that the proposed design can achieve outstanding robustness to common attacks, and it surpasses the existing techniques.
\end{abstract}

Keywords: chaotic maps, robustness, security, video watermarking, wavelet transform. 


\section{Introduction}

$\mathrm{T}$ he growth of the digital multimedia technology and the explosion of data exchanging in the internet have not only enabled distributing of digital data more easily, but also have rewarded the ability of copying it illegally without authorization. A solution to this problem is to add a secure information directly in the content of the digital data. Digital watermarking is such a helpful technique to safeguard patent of the digital multimedia data.

Watermarking is a process that hides data called watermark into multimedia object without corrupting the quality of the object, such that the watermark can be extracted afterward to state the rightful of ownership. The watermark signal can be a pseudo random sequence (Mohammadi, Talebi 2012), a logo image (Behnia etal 2010) or other digital data formats. The watermarking system is expected to be robust to various kinds of malicious and non-malicious attacks. Although some significant progresses have been done recently, one of the major problems in the watermarking algorithms is the absence of security. Chaotic maps have been used to solve this problem. There is a wide interest in using chaotic maps due to their super natural traits. Chaos is identified with non-periodicity and sensitive dependence on initial conditions Alligood etal 2001). We can dedicate initial values as keys in a watermarking system.

All watermarking algorithms can be put in two categories: spatial based watermarking and transform based watermarking. The spatial domain methods modify pixel values directly (Mohanty etal 2007) and (Ye etal 2007) while in the transform domain methods, transform coefficients will be chosen as embedding locations (Ling etal 2011; Wang etal 2010; Liu etal 2010; Cho etal 2010; Phadikar etal 2011).

The proposed watermarking algorithm in (Min-Jeong etal 2012) is a practical real - time video watermarking scheme for high definition video contents. They apply a quantization index modulation technique to low frequency components of full - frame DCT frames calculated by decoding partially compressed videos.

(Wang etal 2010) Presents a fragile watermarking algorithm to authenticate MPEG-4 AVC stream. (Osama etal 2013) has presented a video watermarking based on singular value decomposition which performed in the Discrete Wavelet Transform (DWT) domain.

(Liu etal 2010) Proposes a video watermarking based on discrete Fourier transform (DFT) and Radon transform. They divide the video sequence into groups of pictures, then compute one dimensional DFT along the temporal direction of each groups and finally choose the highest temporal frequencies to embed the watermark in the radon transform of selected frames.

(Masoumi etal 2013) by performing the 3D wavelet transform over the motion part of video, and using sets of pseudo random numbers, the watermark is embedded into 3D wavelet coefficients of some specific sub-bands.

The presented paper proposes a novel video watermarking scheme based on chaotic maps in DWT domain. The watermark signal to be used in our work is encrypted by a chaotic map. In order to have a robust technique, we choose wavelet coefficients of I-frames for embedding locations. Indeed, we apply a 3 level decomposition DWT on I-frames and modify HL3 sub-band coefficients as watermark embedding. Of course we will give more explanation about this selection later. We utilize MPEG-2 standard for compressing the video stream. It is noticeable to say that in our proposed algorithm the embedding stage will be done for a raw video stream (uncompressed video). Therefore our method is independent of type of compression standard.

The reminding of this paper is organized as follows: sections 2 reviews briefly chaotic maps. In section 3 the proposed method is described. Section 4 examines the experimental results. In the last section, we conclude this paper.

\section{CHAOTIC MAPS}


A chaotic orbit is a bounded, non-periodic orbit that displays sensitive Dependence on initial conditions. When we give a precise definition of chaos, we will find that the discussion is simplified if we require a stronger definition of sensitivity, namely that chaotic orbits separate exponentially fast from their neighbors as the map is iterated.

A chaotic orbit is one that forever continues to experience the unstable behavior that an orbit exhibits near a source, but that is not itself fixed or periodic. It never manages to find a sink to be attracted to. At any point of such an orbit, there are points arbitrarily near that will move away from the point during further iteration. This sustained irregularity is quantified by Lyapunov numbers and Lyapunov exponents. We will define the Lyapunov number to be the average per-step divergence rate of nearby points along the orbit, and the Lyapunov exponent to be the natural logarithm of the Lyapunov number. Chaos is defined by a Lyapunov exponent greater than zero.

The significance of the concept of Lyapunov number is that it can be applied to nonperiodic orbits. A characteristic of chaotic orbits is sensitive dependence on initial conditions - the eventual separation of the orbits of nearby initial conditions as the system moves forward in time. In fact, our definition of a chaotic orbit is one that does not tend toward periodicity and whose Lyapunov number is greater than 1 .

There can be chaos in one-dimensional maps, and for invertible maps it can occur in dimensions two and higher. The chaotic map which we use in this study is the Logistic map (Singh etal2010):

$$
x_{n+1}=\rho x_{n}\left(1-x_{n}\right) \text {, }
$$

Where $0<\rho<4$ and $n$ is the iteration number. Initial values and chaotic parameters are the two most significant values recognized as keys in a watermarking algorithm.

\section{THE PROPOSED VIDEO WATERMARKING METHOD}

In this section we explain our proposed DWT video watermarking scheme. Figure. 1, illustrates the block diagram of our method. The watermark signal is a binary logo image. To enjoy more secure algorithm, we encrypt the watermark signal by a chaotic map. Steps of watermark encryption can be summarized as follows:

1. The chaotic map is obtained by a Logistic map by (1). ( $n$, should be selected as same as the length of logo).

Note: here the initial value $x_{0}$, and the chaotic parameter $\rho$, are as keys which should be saved for watermark extraction .

2. The relation (1) is transformed to a binary sequence by (2):

$$
x_{n, \text { binery }}=\text { round }\left(x_{n}\right)
$$

Where $x_{n}$, signifies the constructed sequence by (1) and $x_{n, b i n e r y}$ is the binary sequence.

3. The encrypted watermark is built by (3):

$$
(E w)_{i}=b_{i} \oplus w_{i} \quad i=1,2,3, \ldots, n
$$

Where $b_{i}$ represents the bit of $x_{n, b i n e r y}$ sequence, $w i$ indicates the watermark bit and $n$ is the number of watermark bits. The $\oplus$ operator denotes the 'exclusive or' operator. We call the resulting binary sequence, E-watermark (Ew) which can now be embedded in a digital host video stream.

We devote our method to MPEG-2 standard, but since the embedding procedure is executed on uncompressed video sequences. Thus this method is independent of any compression standard and can be applied to all video compression standards. In MPEG-2 standard [17], each frame is coded as an I, P and B-frame. An I-frame contains only intra coded macro blocks, whereas a P and B-frame can contain either intra or inter coded macro blocks. As we know, compression can be made some alterations on video streams as well as embedding procedure. So we modify only Iframes in the watermark embedding stage: because they have poor compression efficiency (because no temporal prediction is used).

In order to have a robust watermarking algorithm, the embedding is executed in wavelet domain. The steps mentioned bellow can be followed to get the watermarked frame.

1. Select the $Y$ components of I-frames as embedding positions.

2. Apply a three level decomposition 2-D DWT to the $Y$ components. 
Each level decomposition creates 4 subbands, a low frequency sub-band LL and three high frequency sub-bands $\mathrm{LH}, \mathrm{HL}$, and $\mathrm{HH}$ respectively. Figure. 2, shows the HL3 sub-band (gray color) chosen for watermark embedding. This choice is a tradeoff between the invisibility of the watermark and the resilience to attacks. Of course we evaluated this selection by performing some experiments and inferred that this area is more suitable as embedding locations.

3. Modify HL3 coefficients by (4):

$$
H L 3(x, y)=\left\{\begin{array}{l}
H L 3(x, y)-g \\
\quad \text { if }\left(H L 3(x, y)<0 \text { and }(E w)_{i}=0\right) \\
H L 3(x, y)+g \\
\quad \text { if }\left(H L 3(x, y)>0 \text { and }(E w)_{i}=1\right)
\end{array}\right.
$$

where $i=1,2, \ldots, n$.

Where $E w_{i}$ is the E-watermark bit, $n$ is the watermark length, $g$ is the watermark factor and $(x, y)$ s are coefficients' coordinates.

Our try in the embedding stage is to maintain the sign of an HL3coefficient and this is done by initiating the watermark factor " $g$ ". We examined the resistance and transparency of our proposed scheme against changes in the " $g$ " factor. We observed that the best choice for " $g$ " is 5.

Indeed the examination showed that as the watermark factor increases, the PSNR decreases and the robustness of the method increases. Trade off has to be achieved among these two issues.

Note: watermark factor " $\mathrm{g}$ " and the coordinates of the modified coefficients are as watermark keys and they are inevitabilities through watermark extraction process .

4. Apply the three level decomposition inverse 2-D DWT to attain the watermarked frame.

To detect the watermark from a watermarked video stream we require taking the ensuing steps:

1. Separate the $Y$ component of I-frames of watermarked video.

2. Apply a three level decomposition 2-D DWT to the $Y$ components.

3. Separate the HL3 sub-band; therefore name this sub-band as HL3'.

4. Extract watermark signal by (5):
$(E w)_{i}^{\prime}=\left\{\begin{array}{ll}0 & \text { if }\left(H L 3^{\prime}(x, y)<0\right) \\ 1 & \text { if }\left(H L 3^{\prime}(x, y)>0\right)\end{array} \quad, i=1,2, \ldots, n\right.$

Where $(x, y)$ s are coefficients' coordinates with values that have been saved during embedding stage, $n$ is the watermark length, and $(E w)^{\prime}$ is the extracted watermark.

Construct the binary chaotic map by (1) and (2).

Finally, to retrieve the logo image use (6):

$w_{i}^{\prime}=b_{i} \oplus(E w)_{i}^{\prime} \quad i=1,2,3, \ldots, n$

Where $b_{i}$ represents the bit of $x_{n, \text { binery }}$ sequence, $w_{i}^{\prime}$ indicates the extracted watermark bit, and $n$ is the number of watermark bits.

Note: we use $\rho$, and $x_{0}$, values which have been saved as keys in watermark embedding stage.

\section{EXPERIMENTAL RESULTS}

Several tests are used to appraise the performance of this approach in sub-section 4.1. And the comparison of the proposed algorithm with other approaches is carried out in subsection 4.2.

\subsection{Performance analysis}

In order to confirm the performance of our proposed design, it has been tested on several video sequences, such as Foreman $(352 * 288$ resolution) and Rush hour (720*576 resolution). The encrypted watermark is constructed by (1), (2), and (3). The parameters of Logistic map are chosen as $\rho=3.7$ and $x_{0}=0.241$. The watermark signal (Logo) and the encrypted one are shown in Figure. 3, Based on the details in the process of watermark embedding and using (4) watermarked frame has been reached. In Figure. 4, the original host frame and watermarked frame are displayed. Peak Signal to Noise Ratio (PSNR) is utilized in our study to analyze the visual quality of the watermarked frame in contrast with the original frame. PSNR is defined as: 


$$
\begin{aligned}
& P S N R=10 \log \left(\left(255^{\wedge} 2\right) / M S E\right) \\
& M S E=\sum_{i=1}^{M} \sum_{j=1}^{N}\left|X(i, j)-X^{\prime}(i, j)\right|
\end{aligned}
$$

Where $X(i, j)$ is the original signal and $X^{\prime}(i, j)$ is the watermarked one. From Figure. 4, we can perceive that the watermark is not obvious to human observer and both watermarked frame and the original one are close from visual inspection. Table 1, demonstrates the extracted logo for Foreman and Rush hour videos after applying a number of common attacks. In our test Bit Error Rate (BER) measurement is used to calculate the robustness of the proposed solution which is defined as:

$$
B E R=\frac{B}{n}
$$

Where $B$ signifies the number of wrongly detected bits and $n$ is the number of watermark bits. The BER value of the recovered watermark is written below each picture. We also calculate the PSNR between the original and watermarked frame, and the results are brought in Table 1. The less the BER is, the more the vigorous of the planned approach is. When $B E R=0$, it reveals that there is no difference between the original Logo and the extracted one.

Table 1 shows that our design enjoys enhanced immunity against JPEG compression, filtering, noise addition, rotation, and scaling.

In order to evaluate the security of our algorithm, we selected a wrong initial value in watermark extraction stage, Figure. 5, shows a noisy image that means the original logo cannot be extracted and recovered correctly. it's clear that the scheme has extremely sensitive dependence on initial conditions (keys). This study's aim is to get metal flow and distributions of equivalent stress on some special sections such as longitudinal and transverse sections under processing tube tension-reducing.

\subsection{Comparison Results}

To confirm the authority of our method, we compared its implementation, i.e. watermark robustness, against schemes in (Liu etal 2010) and (Osama etal 2013).

Table 2 sums up the performance of method in this study and method in (Liu etal 2010) and (Osama etal 2013), generally. Here comparison is based on Normalized Correlation value which gets by (9):

$$
N C=\frac{\sum_{i}^{j} W(i, j)^{*} W^{\prime}(i, j)}{\sqrt{\sum_{i}^{j} W(i, j)^{2} * \sum_{i}^{j} W^{\prime}(i, j)^{2}}}
$$

Where $w(i, j)$ is the original watermark and $w^{\prime}(i, j)$ is the extracted watermark. It can be observed from Table 2 . The presented method in this study has better robustness compared to (Liu etal 2010) and (Osama etal 2013).

\section{Conclusion}

In this study, we explore a digital watermarking scheme for video sequences. The chaotic watermark is hidden in wavelet coefficients by a special procedure. Our method is robust against several signal processing distortions, such as compression, filtering, noising, and rotation. To improve the security of algorithm the watermark signal is encrypted using a chaotic map. The experimental and comparison results illustrate reasonably good watermark imperceptibility and robustness. 


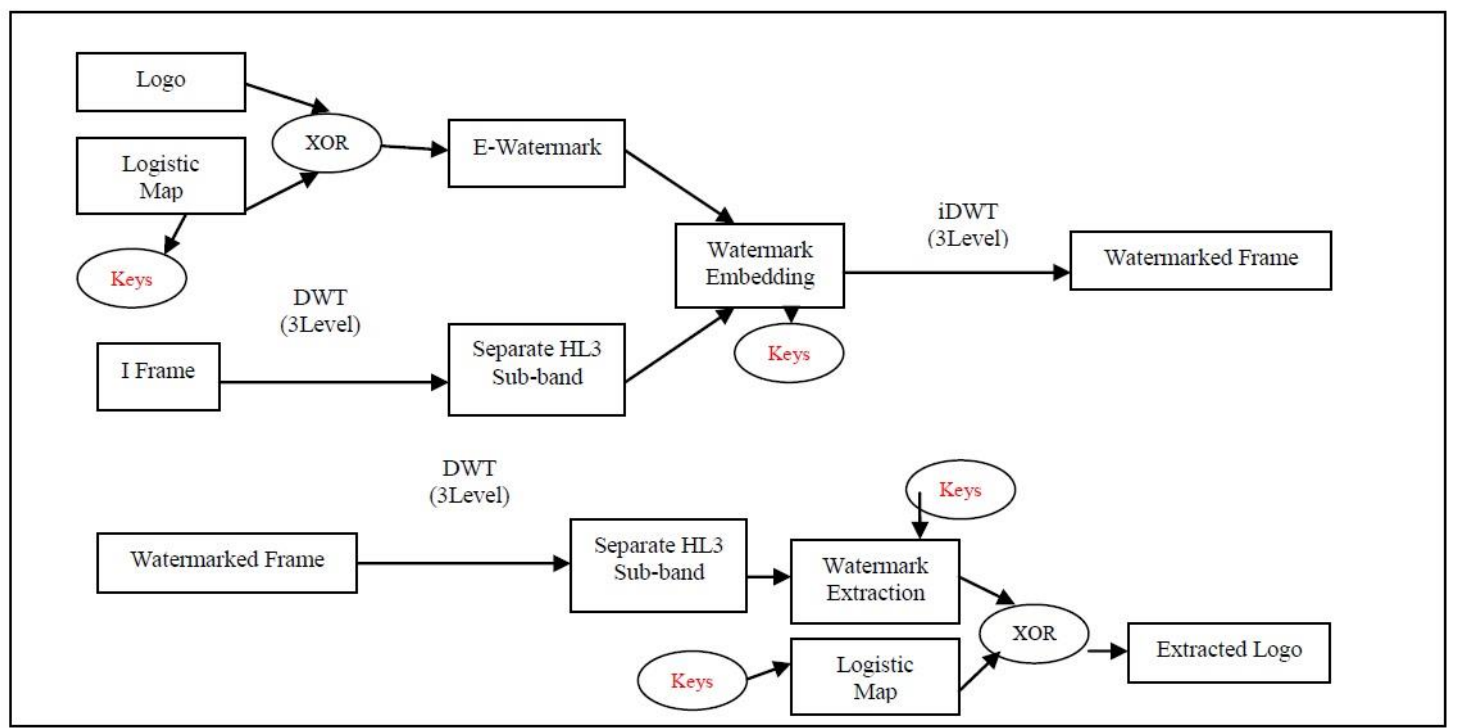

Figure. 1. The block diagram of Watermark Embedding and Watermark Extraction

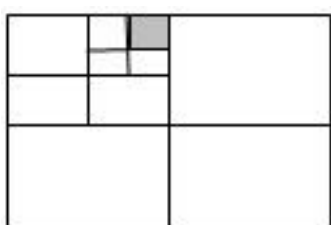

Figure. 2. HL3 sub-band

\begin{tabular}{|c|c|}
\hline WATER & y \\
$\mathrm{a}$ & $\mathrm{b}$ \\
\hline
\end{tabular}

Figure. 3. a) The Watermark Image (Logo). b) The Encrypted Watermark.

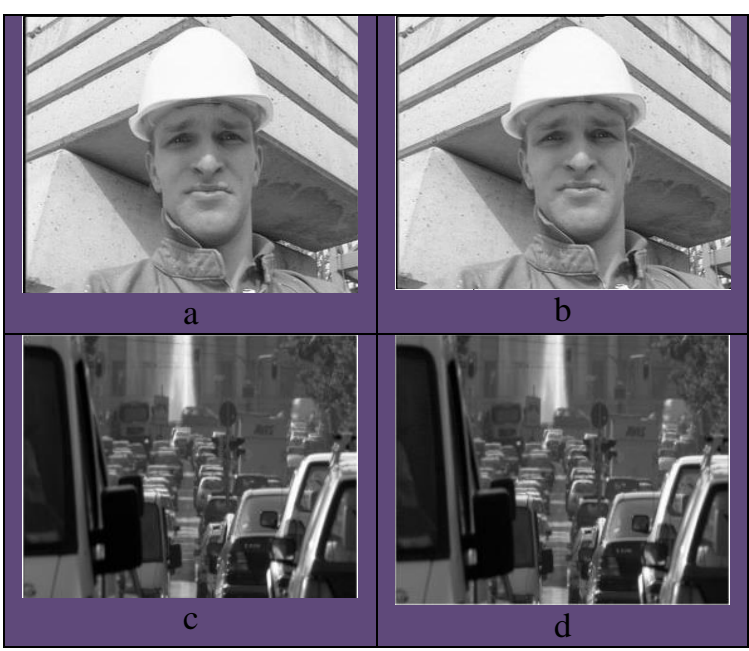

Figure. 4. a) and c) are original frames, b) and d) are watermarked frames.

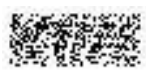

Figure. 5. Wrong extracted watermark(because of selecting a false initial value).
Table 1. Extracted watermark (Logo) after applying

\begin{tabular}{|c|c|c|}
\hline Attacks & $\begin{array}{c}\text { Extracted } \\
\text { Watermark from } \\
\text { Foreman video } \\
\text { stream }\end{array}$ & $\begin{array}{c}\text { Extracted } \\
\text { Watermark from } \\
\text { Rush-hour video } \\
\text { stream }\end{array}$ \\
\hline JPEG (QF=90) & $\begin{array}{c}\text { WATER } \\
\text { BER }=0.00 \\
\text { PSNR }=40.13\end{array}$ & $\begin{array}{c}\text { WATER } \\
\text { BER=0.00 } \\
\text { PSNR }=46.44\end{array}$ \\
\hline JPEG (QF=70) & $\begin{array}{c}\text { W ATER } \\
\text { BER }=0.00 \\
\text { PSNR }=36.4\end{array}$ & $\begin{array}{c}\text { WATER } \\
\text { BER }=0.00 \\
\text { PSNR }=43.63\end{array}$ \\
\hline JPEG (QF=50) & $\begin{array}{c}\text { W A PEP } \\
\text { BER }=0.03 \\
\text { PSNR }=34.79\end{array}$ & $\begin{array}{l}\text { W } \\
\text { BER }=0.17 \\
\text { PSNR }=41.67\end{array}$ \\
\hline $\begin{array}{c}\text { Median Filtering } \\
\text { [3 3] }\end{array}$ & $\begin{array}{l}W \cdot \mathcal{A} T E P \\
\vdots \\
B E R=0.01 \\
\text { PSNR }=35.18\end{array}$ & $\begin{array}{c}\text { WATER } \\
\text { BER }=0.00 \\
\text { PSNR }=44.16\end{array}$ \\
\hline $\begin{array}{c}\text { Median Filtering } \\
\qquad[55]\end{array}$ & $\begin{array}{l}\text { WEA } \\
\text { BER=0.04 } \\
\text { PSNR=31.57 }\end{array}$ & $\begin{array}{c}\text { WATER } \\
\text { BER }=0.00 \\
\text { PSNR }=38.58\end{array}$ \\
\hline $\begin{array}{l}\text { Gaussian } \\
\text { Filtering }\end{array}$ & $\begin{array}{c}\text { W ATER } \\
\text { BER }=0.00 \\
\text { PSNR }=39.33\end{array}$ & $\begin{array}{c}\text { WATER } \\
\text { BER }=0.00 \\
\text { PSNR }=47.4\end{array}$ \\
\hline & WATER & WHTER \\
\hline $\begin{array}{l}\text { Salt\& Pepper } \\
\quad(0.001)\end{array}$ & $\begin{array}{c}\text { BER }=0.00 \\
\text { PSNR }=35.12\end{array}$ & $\begin{array}{c}\text { BER }=0.02 \\
\text { PSNR }=35.11\end{array}$ \\
\hline & WATER & WATER \\
\hline Rotation $\left(0.25^{\circ}\right)$ & $\begin{array}{l}\text { BER }=0.00 \\
\text { PSNR }=31.53 \\
W \text { STER. }\end{array}$ & 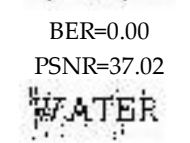 \\
\hline
\end{tabular}




\begin{tabular}{|c|c|c|}
\hline \multirow{3}{*}{ Rotation $\left(10^{\circ}\right)$} & $\begin{array}{c}\text { BER }=0.02 \\
\text { PSNR }=1788\end{array}$ & $\begin{array}{c}\text { BER }=0.05 \\
\text { PSNR }=23.97\end{array}$ \\
\hline & H.STEP & 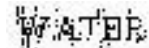 \\
\hline & $\mathrm{BER}=0.03$ & $\mathrm{BER}=0.08$ \\
\hline \multirow{3}{*}{ Scaling (0.5) } & $\begin{array}{l}\text { PSNR }=14.79 \\
\text { W ATER }\end{array}$ & $\begin{array}{l}\text { PSNR=21.26 } \\
\text { WATER }\end{array}$ \\
\hline & $\mathrm{BER}=0.00$ & $\mathrm{BER}=0.00$ \\
\hline & $\mathrm{PSNR}=30.26$ & $\mathrm{PSNR}=42.79$ \\
\hline
\end{tabular}

Table 2. comparison of Normalized Correlation values for our scheme and [8] and [13].

\begin{tabular}{cccc}
\hline Attacks & $\begin{array}{c}\text { Our proposed } \\
\text { method }\end{array}$ & $\begin{array}{c}\text { Osama etal } \\
2013\end{array}$ & Liu etal 2010 \\
\hline${\text { Rotation } \mathbf{5}^{\circ}}^{\circ}$ & 96 & 100 & 73.22 \\
Rotation $\mathbf{1 0}^{\circ}$ & 94 & 88 & 73.11 \\
Rotation $\mathbf{1 5}^{\circ}$ & 92 & 65 & 73.83 \\
\hline
\end{tabular}

\section{References}

Alligood, K. T., Sauer, Tim D., and Yorke, James A. (2001). Chaos: An Introduction to Dynamical systems. Springer, New York.

Behnia, S., Teshnelab, M., and Ayubi, P. (2010). Multiple-watermarking schem based on improved chaotic maps. Commun Nonlinear Sci Numer Simulat 15, pp. 2469-2478.

Cho, D., Do, H., Choi, H., and Kim, T. (2010). A blind MPEG-2 video watermarking robust to camcorder recording. Signal Process (Elsevier). 90:1327-32.

Ghanbari, M. (2003).IEE Standard Codecs Image Compression To Advanced Video Coding eBook-LiB.

Ling, H., Wang, L., Zou, F., Lu, Z., and Li, P. (2011). Robust video watermarking based on affine invariant regions in the compressed domain. Signal Process (Elsevier).91:1863-75.

Liu, Y., and Zhao, J.(2010). A new video watermarking algorithm based on 1D DFT and Radon transform. Signal Process (Elsevier). 90:626-39.

Masoumi, M., and Amiri, S. (2013). A blind scene-based watermarking for video copyright protection," Int. J. Electron. Commun. (AEU) 67. 528-535.

Min-Jeong Lee, Dong-Hyuck Im, Hae-Yeoun Lee, Kyung-Su Kim, Heung-Kyu Lee. (2012). Realtime video watermarking system on the compressed domain for high-definition video contents: practical issues. Digital Signal Processing 22. 190-198.

Mohammadi, S., Talebi, S. (2012). An Image watermarking algorithm based on chaotic maps and wavelet transform. $9^{\text {th }}$ International ISC Conference.

Mohanty. SP., Kougianos, E., and Ranganathan, N. (2007). VLSI architecture and chip for combined invisible robust and fragile watermarking. IET Comput Digital Tech (CDT ),1(5):600-11.

Osama S, Faragallah. (2013). Efficient video watermarking based on singular value decompositon in the discrete wavelet transform domain. Int. J. Electron. Commun. (AEU) 67. 189-196.

Phadikar, A., Maity, SP., and Verma, B. (2011). Region based QIM digital watermarking scheme for image database in DCT domain. Comput Electr Eng (Elsevier ). 37:339-55.

Radu O. Preda and Dragos N. Vizireanu. (2010). A robust digital watermarking schem for video copyright protection in the wavelet domain. Measurement 43. 1720-1726.

Singh, N., and Sinha, A. (2010). Digital image watermarking using gyrator transform and chaotic maps," Optic 121, pp. 1427-1437.

Wang, C.C., and Hsu, Y.C. (2010). Fragile watermarking scheme for H.264 video stream authentication. Optical Engineering 49 (2).

Wang, X., Yang, Y., and Yang, H. (2010). Invariant image watermarking using multiscale Harris detector and wavelet moments. Comput Electr Eng (Elsevier).36:31-44.

Ye, D., Zou, C., Dai, Y., and Wang, Z. (2007). A new adaptive watermarking for real-time MPEG videos. Appl Math Comput (Elsevier);185:907-18. 